\title{
Preliminary investigation of the role of BTB domain-containing 3 gene in the proliferation and metastasis of hepatocellular carcinoma
}

\author{
WEIWEI XIAO ${ }^{1}$, WEI ZHAO ${ }^{1}$, LING LI ${ }^{1}$, QINGHUA WU ${ }^{1}$, LI ZHU ${ }^{1}$, \\ QIWEI ZHANG ${ }^{1}$, WEIPENG DAI ${ }^{1}, \mathrm{YU} \mathrm{WANG}^{2 *}$ and BAO ZHANG ${ }^{1 *}$ \\ ${ }^{1}$ Biosafety Level-3 Laboratory, Guangdong Provincial Key Laboratory of Tropical Disease Research, \\ School of Public Health; ${ }^{2}$ Department of Hepatobiliary Surgery, Nanfang Hospital, \\ Southern Medical University, Guangzhou, Guangdong 510515, P.R. China
}

Received September 23, 2015; Accepted April 6, 2017

DOI: $10.3892 / \mathrm{ol} .2017 .6369$

\begin{abstract}
Hepatocellular carcinoma (HCC) is one of the leading digestive malignancies, with a high metastasis and recurrence. The development and rapid progression of HCC involves numerous complex molecular and cellular events. Therefore, developing effective methods for the prevention and treatment of HCC requires an improved understanding of the biological development of HCC. In our previous analysis of the tissue microarray data, the BTB domain-containing 3 (BTBD3) gene was upregulated in HCC tissues, indicating that it may be a cancer-associated gene and serve a role in the occurrence and development of HCC. In the present study, reverse transcription-quantitative polymerase chain reaction and western blotting were performed to analyze the expression level of BTBD3 in four HCC cell lines; HepG2, Huh7, Bel7404 and Hep3B. The overexpression of BTBD3 in the four cell lines confirmed that BTBD3 was a cancer-associated gene. Subsequently, a short interfering RNA interference technique was performed to investigate the effect of BTBD3 expression
\end{abstract}

Correspondence to: Dr Bao Zhang, Biosafety Level-3 Laboratory, Guangdong Provincial Key Laboratory of Tropical Disease Research, School of Public Health, Southern Medical University, 1838 Baiyun Avenue North, Guangzhou, Guangdong 510515, P.R. China

E-mail: zhang20051005@126.com

Dr Yu Wang, Department of Hepatobiliary Surgery, Nanfang Hospital, Southern Medical University, 1838 Baiyun Avenue North, Guangzhou, Guangdong 510515, P.R. China

E-mail:wyjxq163@163.com

*Contributed equally

Abbreviations: BTBD3, BTB domain-containing 3; HCC, hepatocellular carcinoma

Key words: hepatocellular carcinoma, BTB domain-containing 3, invasion, metastasis on the proliferation and metastasis of Bel7407 cells. MTS assay and flow cytometry were used to evaluate the effect of BTBD3 on the proliferation and cell cycle, and a scratch test and Transwell assay were performed to determine the alterations to the migration and invasion of cancer cells. The results revealed that there was a minimal impact on cell proliferation following silencing of the BTBD3 gene. However, significant inhibition of cell invasion was demonstrated in the scratch test and the Transwell model. Based on these results, it was suggested that BTBD3 gene may be overexpressed in HCC tissues and cell lines, which promotes the invasion and metastasis of cancer cells without affecting cell proliferation.

\section{Introduction}

Liver cancer is one of the most commonly occurring malignant tumors; its global incidence and mortality rate are ranked 5th and 3rd among malignant tumors, respectively $(1,2)$. The majority of cases of liver cancer are hepatocellular carcinoma (HCC) (3). The global distribution of HCC is disproportional, with the highest incidence reported in Asia and Sub-Saharan Africa, particularly in China (3). Patients with HCC exhibit an overall 5-year survival rate of only 5\% (4). In total, $70 \%$ of patients experience relapse within five years of undergoing surgery and $>80 \%$ of recurrences are within the remaining liver tissue (5). Patients with HCC often exhibit various outcomes, even when identical clinicopathological features are observed; this suggests that the development and rapid progression of HCC involves numerous complex molecular and cellular events (6). Therefore, developing effective methods for the prevention and treatment of HCC requires an improved understanding of the biological development of HCC.

As demonstrated in our previous analysis of the tissue microarray data, the BTB domain-containing 3 (BTBD3) gene was upregulated in $\mathrm{HCC}$ tissues, indicating that it may be a cancer-associated gene and have a role in the occurrence and development of HCC. The present study aimed to further analyze the expression levels of BTBD3 in HCC cell lines and explore its role in the occurrence, development and metastasis of HCC. 


\section{Materials and methods}

Cell line and cell culture. The human immortalized normal hepatocyte LO2 cell line, and HCC HepG2, Huh7, Bel7404 and Hep3B cell lines (Type Culture Collection of the Chinese Academy of Sciences, Shanghai, China), were maintained in Dulbecco's modified Eagle's medium (Gibco; Thermo Fisher Scientific, Inc., Waltham, MA, USA) supplemented with 10\% fatal bovine serum (Gibco; Thermo Fisher Scientific, Inc.), $100 \mathrm{U} / \mathrm{ml}$ penicillin and $100 \mu \mathrm{g} / \mathrm{ml}$ streptomycin (Sigma-Aldrich; Merck KGaA, Darmstadt, Germany). The cells were incubated at $37^{\circ} \mathrm{C}$ in $5 \% \mathrm{CO}_{2}$.

Bioinformatics analysis. The HCC tissue microarray data of GSE14215 and GSE29217 were downloaded from the Gene Expression Omnibus database of the National Centre for Biotechnology Information (https://www.ncbi.nlm.nih .gov/geo/). The genes with common various expressions were analyzed using Genespring version 11.0 software (Agilent Technologies, Inc., Santa Clara, CA, USA) and determined using meta-analysis.

Reverse transcription-quantitative polymerase chain reaction (RT-qPCR) analysis. Total RNA from all the cell lines was isolated using miRNeasy Mini kit (Qiagen $\mathrm{GmbH}$, Hilden, Germany) according to the manufacturer's instructions. The RT-qPCR amplification for the quantification of the BTBD3 and GAPDH mRNAs was performed using an ABI PRISM 7500 Sequence Detection System (Applied Biosystems; Thermo Fisher Scientific, Inc.) and a SYBR ${ }^{\circledR}$ Premix Ex Taq ${ }^{\mathrm{TM}}$ (Tli RNaseH Plus) kit (Takara Bio, Inc., Otsu, Japan). The following primers were used: BTBD3 sense, 5'-TGGCAGATGTACATT TTGTGG-3' and antisense, 5'-AACACAGAGCTCCCAACA GC-3'; GAPDH sense, 5'-GGGAAACTGTGGCGTGATG-3' and antisense, 5'- GAGTGGGTGTCGCTGTTGA-3'. The RT reaction was performed at $42^{\circ} \mathrm{C}$ for $30 \mathrm{~min}$ and then $70^{\circ} \mathrm{C}$ for $15 \mathrm{sec}$. qPCR was performed using the cDNA as template under the following conditions: PCR initial activation at $95^{\circ} \mathrm{C}$ for $15 \mathrm{~min}$, followed by 40 cycles at $94^{\circ} \mathrm{C}$ for $15 \mathrm{sec}, 55^{\circ} \mathrm{C}$ for $30 \mathrm{sec}$ and $70^{\circ} \mathrm{C}$ for $30 \mathrm{sec}$. The expression level of BTBD3 was normalized as relative expression to GAPDH. Relative expression was calculated as $2^{-\Delta \Delta C q}(7)$. Each PCR reaction was performed in triplicate.

Western blot analysis. Total proteins were extracted from LO2 and HepG2, Huh7, Bel7404 and Hep3B cell lines. Equal amounts of protein $(50 \mu \mathrm{g})$ were loaded for electrophoreses on $10 \%$ SDS-PAGE gels, transferred to polyvinylidene fluoride membrane and incubated overnight at $4^{\circ} \mathrm{C}$ with the appropriate primary antibodies as follows: Monoclonal rabbit anti-human BTBD3 (dilution, 1:1,500; catalog no. ABIN1398849; Abcam, Cambridge, MA, USA) and GAPDH (dilution, 1:10,000; catalog no. G8140; US Biological, Swampscott, MA, USA). Following incubation with the horseradish peroxidase-conjugated bovine monoclonal anti-rabbit secondary antibody (dilution, 1:5,000; catalog no. COL18A1; Boster Biological Technology, Pleasanton, CA, USA) for $2 \mathrm{~h}$ at room temperature, the immumoreactive proteins were visualized using the enhanced chemiluminescence (GE Healthcare, Chicago, IL, USA) method. The amounts of proteins were quantified by scanning densitometry using Quantity One software (Bio-Rad Laboratories, Inc., Hercules, CA, USA) and normalized to the relative internal standards by GAPDH protein band density.

Short interfering (si)RNA transfection. As presented in Table I, two BTBD3 siRNAs, siRNA-79 and siRNA-81, and a negative control siRNA, siRNA-negative control (NC), were designed and synthesized by Sigma-Aldrich (Merck KGaA). Using Lipofectamine RNAiMAX (Invitrogen; Thermo Fisher Scientific, Inc.), siRNA-79, siRNA-81 and siRNA-NC were transfected into Bel7404 cells, according to the manufacturer's instructions. A total of 24-h after transfection, the interference efficiency of siRNA was determined by RT-qPCR assay.

MTS assay. Bel7407 cells were seeded into 96-well plates at a density of $1 \times 10^{4}$ cells/well and cultivated at $37^{\circ} \mathrm{C}$ in $5 \% \mathrm{CO}_{2}$ for $24 \mathrm{~h}$. Subsequently, Bel 7407 cells were transfected at $37^{\circ} \mathrm{C}$ for $6 \mathrm{~h}$ with BTBD3 siRNA-79 ( $25 \mathrm{nM})$ and siRNA-NC ( $25 \mathrm{nM})$ using Lipofectamine RNAiMAX (Invitrogen; Thermo Fisher Scientific, Inc.), respectively. MTS (1:10 dilution) was added to the cells every $24 \mathrm{~h}(0,24,48$ and $72 \mathrm{~h})$, which were then incubated for $4 \mathrm{~h}$ at $37^{\circ} \mathrm{C}$. The optical density of the culture medium at $490 \mathrm{~nm}$ was evaluated using an EnVision plate reader (PerkinElmer, Inc., Waltham, MA, USA). Triplicate wells were analyzed for each assay.

Flow cytometry. Bel 7404 cells seeded at a density of $5 \times 10^{6}$ per well into 6-well plates were transfected with siRNA-79 $(25 \mathrm{nM})$ or siRNA-NC $(25 \mathrm{nM})$. A total of 48 -h after transfection, cells were harvested, fixed with $70 \%$ ethanol and then resuspended in propidium iodide/RNase Staining Buffer (BD Biosciences, Franklin Lakes, NJ, USA). The DNA content of cells was analyzed using a MoFlo XDPCell Sorter (Beckman Coulter, Inc., Brea, CA, USA). The cell numbers in each phase of the cell cycle was determined using FlowJo software (FlowJo version 7.6.3, LLC, Ashland, OR, USA).

Wound healing assays. Bel7404 cells were grown to 90\% confluence in the 6-well flat-bottomed plates. The monolayer cells were scratched with a sterile $200-\mu 1$ pipette tip to create a denuded zone (gap) of $1 \mathrm{~mm}$ width. The remaining cells were washed twice with PBS to remove cell debris and incubated at $37^{\circ} \mathrm{C}$ in DMEM/F12 (Gibco; Thermo Fisher Scientific, Inc.). The scratched areas were imaged at 0, 6, 24 and $48 \mathrm{~h}$ after wounding using a phase-contrast microscope (Leica Micro-systems GmbH, Wetzlar, Germany, magnification, $\mathrm{x} 200)$. Cell motility was evaluated as percentages of cell coverage to the initial cell-free zone using ImageJ software (Image J version 2.1.4.7, National Institutes of Health, Bethesda, MA, USA). The cell mobility rate was evaluated as follows: 1-distance at various time points/distance at $0 \mathrm{~h}$ )x $100 \%$ Three randomly selected wound areas were analyzed.

Transwell invasion assay. Following siRNA transfection, $1 \times 10^{4}$ Bel7404 cells resuspended in serum-free DMEM/F12 (Gibco; Thermo Fisher Scientific, Inc.) were seeded in the upper chambers, and $600 \mu \mathrm{l}$ complete DMEM medium (Gibco; Thermo Fisher Scientific, Inc.) was added to the lower chambers. Following incubation at $37^{\circ} \mathrm{C}$ for $18 \mathrm{~h}$, the non-migrated cells on the upper layer were removed using cotton swabs and 
Table I. BTB domain-containing 3 siRNA sequences.

\begin{tabular}{lc}
\hline Strand & Sequence (5'-3') \\
\hline siRNA-79 & \\
Sense & 5'-CUUAGCUCAUCUGCAAAUAdTdT-3' \\
Antisense & 5'-UAUUUGCAGAUGAGCUAAGdTdT-3' \\
Target & 5'-AUAAACGUCUACUCGAUUC-3' \\
siRNA-81 & \\
Sense & 5'-CCAGUUUGCAGUUGAUAAAdTdT-3' \\
Antisense & 5'-UUUAUCAACUGCAAACUGGdTdT-3' \\
Target & 5'-AAAUAGUUGACGUUUGACC-3' \\
siRNA-NC & \\
Sense & 5'-UUCUCCGAACGUGUCACGUdTdT-3' \\
Antisense & 5'-ACGUGACACGUUCGGAGAAdTdT-3' \\
Target & 5'-UGCACUGUGCAAGCCUCUU-3' \\
\end{tabular}

siRNA, short interfering RNA; NC, negative control.

the penetrating cells in the lower layer were fixed in $4 \%$ paraformaldehyde for $15 \mathrm{~min}$ and stained with $0.1 \%$ crystal violet for $10-20 \mathrm{~min}$ at room temperature. The cells in five random microscopic fields were counted and imaged using a light microscope with a DP70CCD system (Olympus Corp., Tokyo, Japan). All experiments were performed in triplicate.

Statistical analysis. All statistical analyses were performed using SPSS (version 17.0; SPSS Inc., Chicago, IL, USA). Data are presented as the mean \pm standard deviation from three independent experiments and statistical analyses were performed using Student's t-test. $\mathrm{P}<0.05$ was considered to indicate a statistically significant difference.

\section{Results}

Analysis of the expression level of the BTBD3 in HCC tissues using microarray data. Gene microarray data of GSE14215 and GSE29217 were downloaded from NCBI and analyzed using Genespring software. Compared with normal tissues, the analysis results demonstrated that 227 and 281 genes were upregulated and downregulated, respectively, in the HCC tissues. Among the upregulated genes, BTBD3 ranked 60th, with 2.45-fold upregulation, indicating its potential role in the occurrence and development of HCC.

Expression levels of BTBD3 in HCC cell lines. The expression levels of BTBD3 in HCC HepG2, Huh7, Bel7407 and Hep3B cell lines and liver normal LO2 cell line were determined by real-time RT-qPCR and western blotting. The expression levels of BTBD3 mRNA in the HCC cell lines were significantly increased compared with LO2 cells. As presented in Fig. 1A, compared with LO2 cells, expression was upregulated 5.3, 1.8, 2.5 and 2.6-fold in HepG2, Huh7, Bel7404 and Hep3B cells, respectively. The results of the western blot analysis confirmed that the expression levels of BTBD3 protein in HCC cell lines was significantly increased compared with LO2 cells (Fig. 1B and $\mathrm{C}$ ), which was consistent with the findings of RT-qPCR.
A

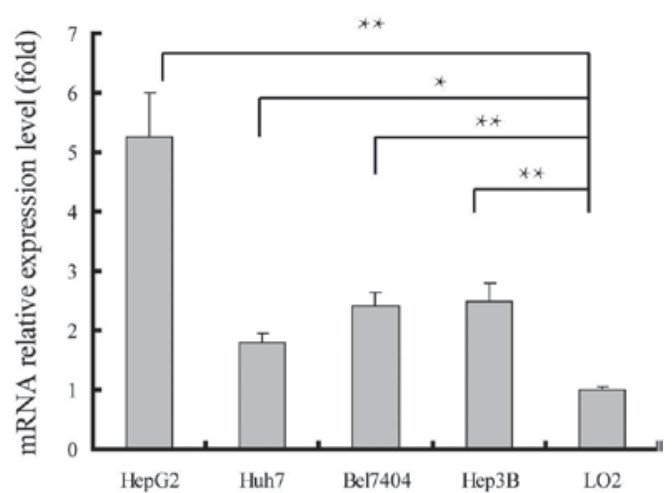

$\mathbf{B}$

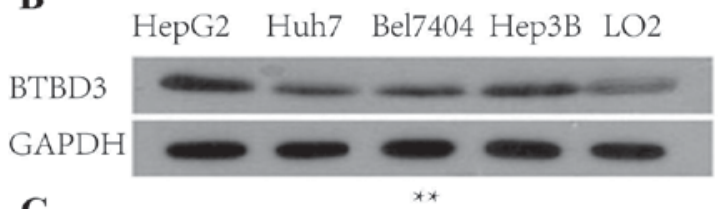

C

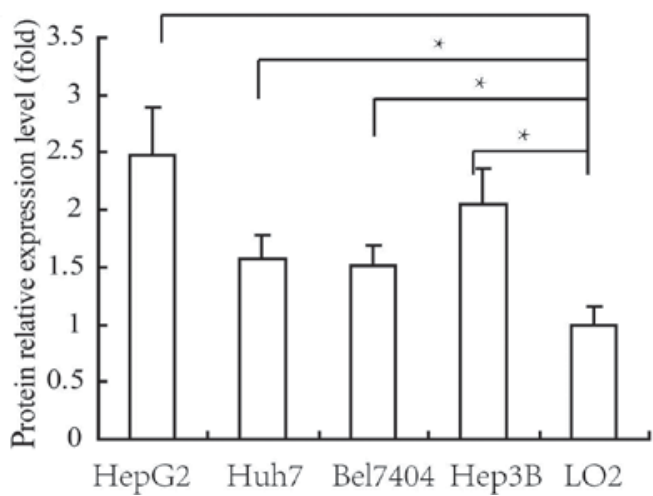

Figure 1. BTBD3 expression levels in HCC cell lines. (A)The expression levels of BTBD3 mRNA in four HCC cell lines and LO2 liver normal cell lines were analyzed by reverse transcription-quantitative polymerase chain reaction. (B) The expression levels of BTBD3 protein in four $\mathrm{HCC}$ cell lines and the liver normal LO2 cell line were analyzed by western blotting. (C) Scanning densitometry analysis of amounts of protein. ${ }^{*} \mathrm{P}<0.05,{ }^{* *} \mathrm{P}<0.01$. HCC, hepatocellular carcinoma; BTBD3, BTB domain-containing 3 .

Therefore, it was suggested that overexpression of BTBD3 gene was associated with the proliferation or/and metastasis of $\mathrm{HCC}$.

Effect of BTBD3 siRNA interference on cell proliferation. To determine the effects of BTBD3 expression on cell growth and migration, two BTBD3 siRNA sequences (siRNA-79 and siRNA-81) and a negative control siRNA-NC were designed, synthesized and transfected into Bel7407 cells. As demonstrated in by RT-qPCR, $24 \mathrm{~h}$ after siRNA transfection, significant interference, with $70 \%$ downregulation of the BTBD3 gene, was observed at $25 \mathrm{nM}$ of siRNA-79 and siRNA-81, and therefore $25 \mathrm{nM}$ siRNA-79 was selected for use in the following experiments (Fig. 2A).

The present study analyzed cell proliferation of Bel7407 cells upon silencing of BTBD3 by MTS assay (Fig. 2B). Compared with at $0 \mathrm{~h}$, a 136, 227 and 398\% increase in cell growth was observed in the negative control group at 24, 48 and $72 \mathrm{~h}$ after transfection, respectively, whereas a 127,192 and $320 \%$ increase was observed at 24,48 and $72 \mathrm{~h}$ after transfection, respectively, in the BTBD3 siRNA-79 group. 


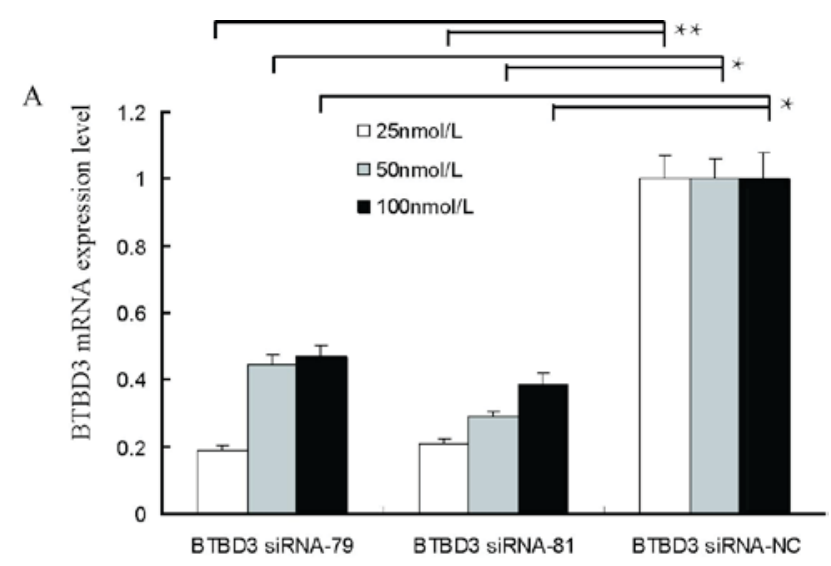

B
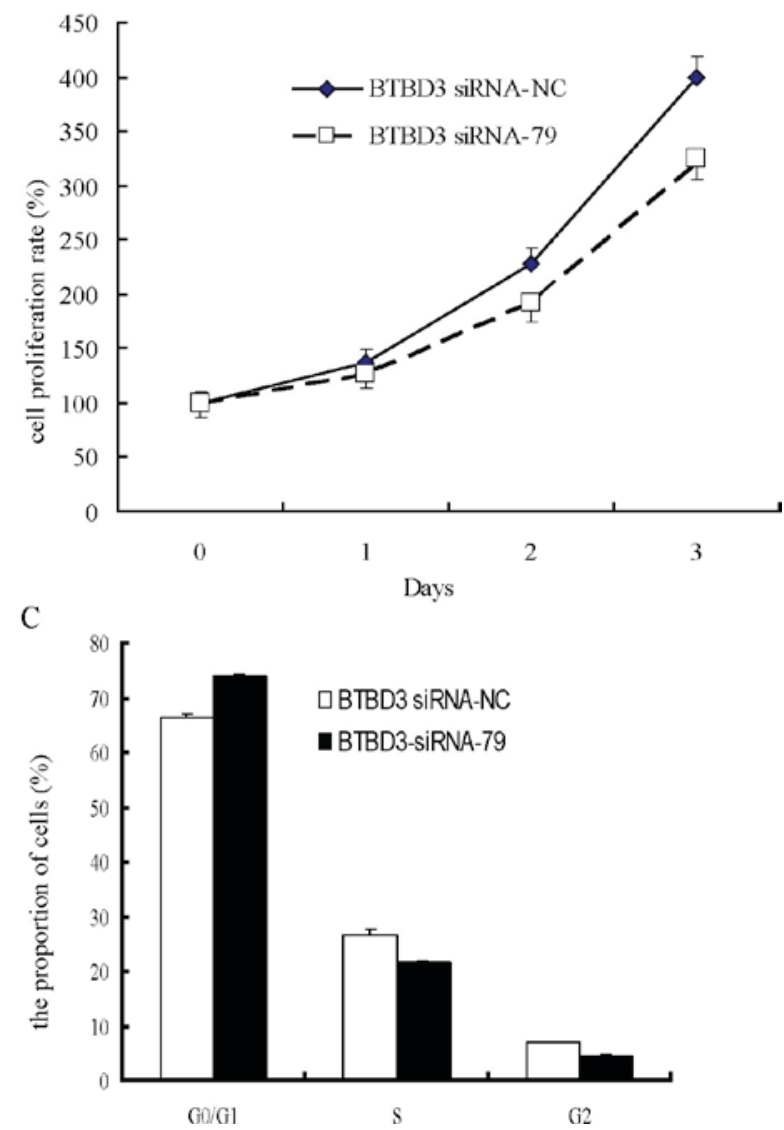

Figure 2. Effects of BTBD3 silencing on cell proliferation and cell cycle distribution were determined by MTS assay and flow cytometry. (A) Inhibition efficiency of various BTBD3 siRNA molecules with various concentrations. (B) Effects of BTBD3 silencing on cell proliferation was evaluated by MTS assay in Bel7407 cells over the time course of transfection. No significant difference was observed between the BTBD3 siRNA-79 group and the control. (C) Cell cycle analysis was performed by flow cytometry for Bel7407 cells at 48-h post-transfection. No significant difference in the percentage of cells in the $G_{0} / G_{1}, G_{2}$ and $S$ cell cycle stages were observed between the BTBD3 siRNA-79 group and control $\left({ }^{*} \mathrm{P}>0.05\right) .{ }^{*} \mathrm{P}<0.05$, ${ }^{* *} \mathrm{P}<0.01$. siRNA, short interfering RNA; NC, negative control; BTBD3, BTB domain-containing 3.

No significant difference was observed between the BTBD3 siRNA-79 group and the negative control $(\mathrm{P}>0.05)$, indicating a lack of association between the BTBD3 gene and cell proliferation.

Flow cytometry was performed to determine the change in cell cycle of Bel7407 cells at $48 \mathrm{~h}$ post-transfection (Fig. 2C).

\section{A}

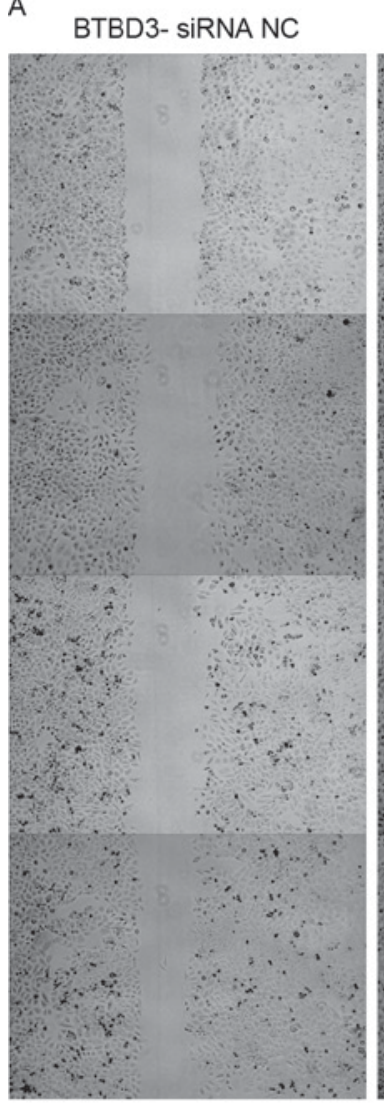

BTBD3- siRNA-79

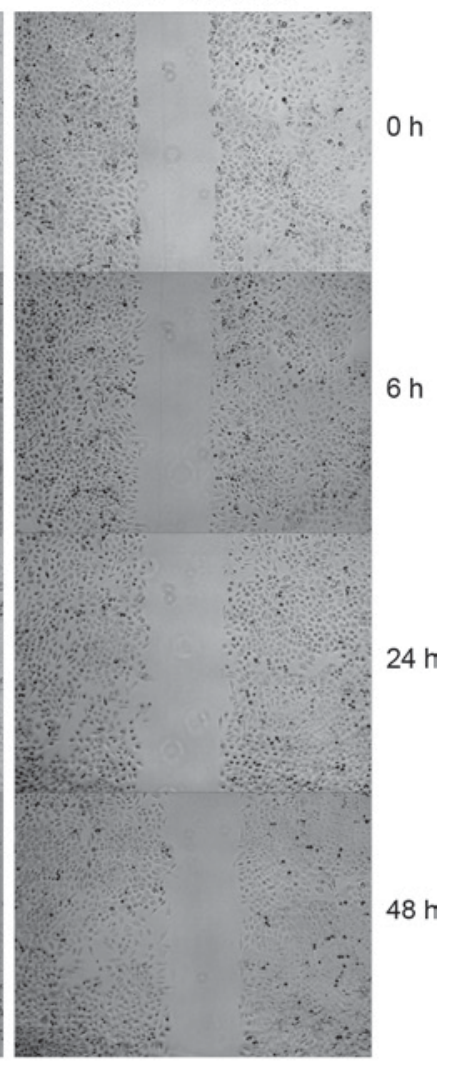

B

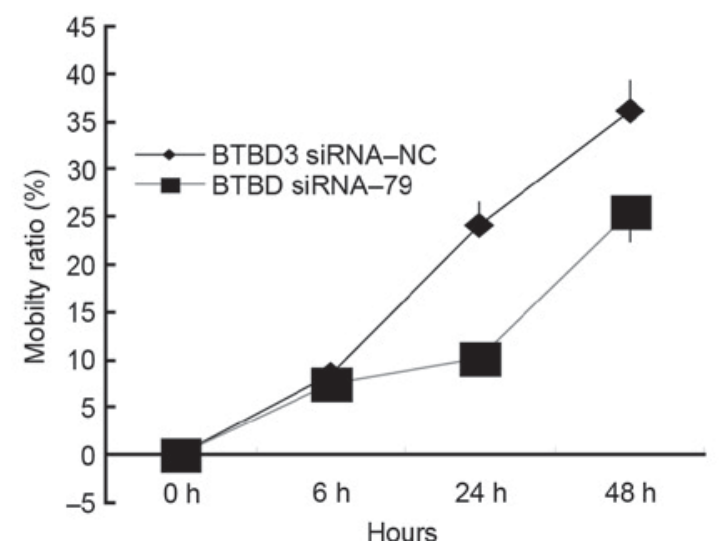

Figure 3. The motility of Bel7404 cells was determined by cell wound assay. (A) Images of the scratched areas were captured using a phase-contrast microscope at 0, 6, 24 and 48-h after wounding (magnification, $\mathrm{x} 200$ ). The relative width of the wounds in the siRNA-79 group was significantly wider compared with in the control siRNA-NC group. (B) The cell mobility rate of the Bel7404 cells was decreased in the siRNA-79 group compared with in the control siRNA-NC group. siRNA, short interfering RNA; NC, negative control; BTBD3, BTB domain-containing 3.

No significant difference in the percentage of cells in the $\mathrm{G}_{0} / \mathrm{G}_{1}$, $\mathrm{G}_{2}$ and $\mathrm{S}$ stages of the cell cycle were observed between the BTBD3 siRNA-79 group and negative control, which further confirmed that BTBD3 did not promote cell proliferation.

Effect of BTBD3 siRNA interference on cell migration and invasion. In order to investigate of the role of BTBD3 gene in the metastasis of HCC, a cell scratch test and a Transwell assay were performed to determine the migration and invasion of Bel7407 cells following siRNA transfection. 

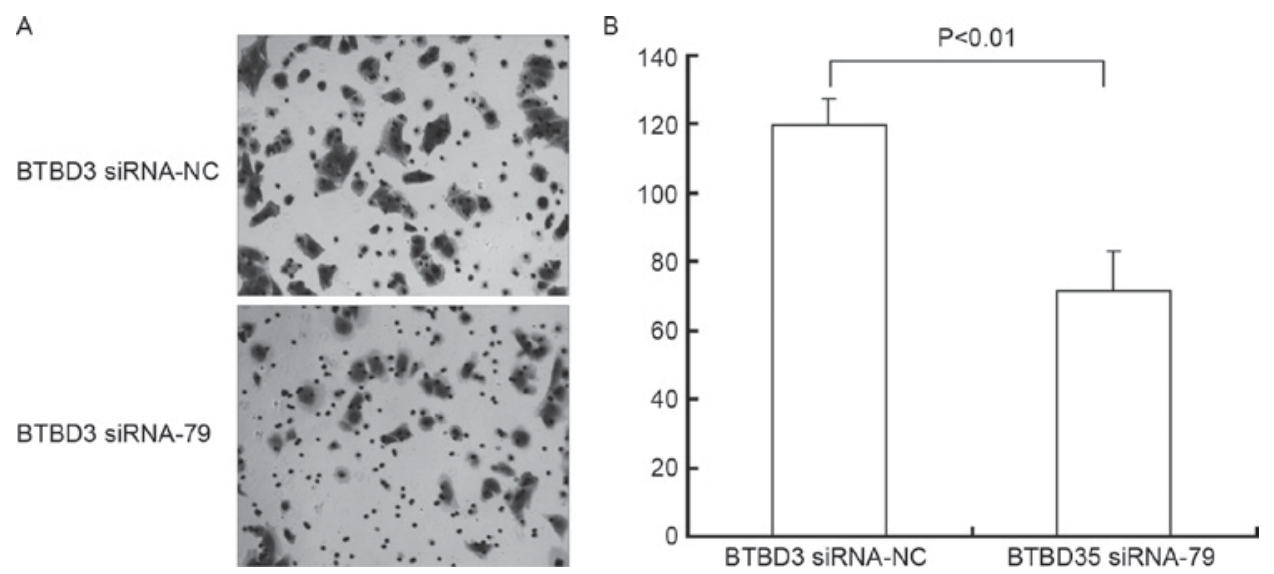

Figure 4. The migration and invasion of Bel7404 cells was determined by Transwell chamber assay. (A) Images of the Bel7404 cells that invaded through the Transwell membrane (magnification, x200). (B) The number of invading Bel7404 cells decreased significantly in the siRNA-79 group $(\mathrm{P}<0.01)$ compared with the siRNA-NC group. siRNA, short interfering RNA; NC, negative control; BTBD3, BTB domain-containing 3.

As demonstrated by the cell scratch test (Fig. 3A), 6, 24 and $48 \mathrm{~h}$ after initiation, the relative widths of the scratches in the control group were 91,76 and $63 \%$ of the original width, respectively. The relative widths in the siRNA-79 group were 93, 90 and $76 \%$, respectively, which were significantly wider than those of the control group (Fig. 3B; $\mathrm{P}=0.0361$ ). These results indicated that BTBD3 gene silencing resulted in a decreased capacity for cell migration in Bel7404 cells.

As demonstrated in the Transwell chamber assay, the migration of Bel7407 cells was significantly inhibited (Fig. 4A) following siRNA transfection. As presented in Fig. 4B, the number of invasive and metastatic cells in the siRNA-NC control group was $119.5 \pm 7.31$, which was significantly higher $(\mathrm{P}=0.007)$ than the BTBD3 siRNA-79 group $(71.33 \pm 11.27)$.

\section{Discussion}

HCC is extremely malignant and highly invasive, with a high incidence of both intra-hepatic and extra-hepatic metastases. Metastatic recurrence is the most important reason for the unsatisfactory prognosis following surgery $(4-6,8,9)$. Owing to its high incidence, metastasis and mortality rates, HCC has attracted attention for the investigation of the mechanism underlying its occurrence and development. With the development of molecular biology techniques, including high-throughput detection technology (microarray) and detailed sequencing techniques, there has been great progress in the elucidation of the mechanisms underlying HCC (10-12). Approximately 200 genes have been associated with the proliferation, metastasis and recurrence of HCC (11).

BTBD3 is located at 20p12.2, with two splice variants coding for 482 and 385 amino acids, respectively. Within this gene, there is a BTB (broad-complex, tramtrack and bric-à-brac) structural domain (amino acids 113-219), a nuclear localization signal region (amino acids 55-70), and a structural domain containing BACK (amino acids 226-335) and PHR (amino acids 226-335) regions. At present, there have been limited studies investigating the function of the BTBD3 gene (13-20). A previous study by Zhang et al (13), investigating the function of hsa-let-7i in colon cancer metastasis, is the only study to have suggested the BTBD3 gene may be the target of hsa-let-7i. The analysis of the microarray data of gene expression levels in HCC tissues in the present study revealed that the BTBD3 gene was upregulated by 2.45 -fold in cancer tissues, which indicated the potential role of the BTBD3 gene in the occurrence and development of HCC. However, to the best of our knowledge, no previous studies have investigated the association between the BTBD3 gene and HCC.

In order to investigate the role of the BTBD3 gene in the occurrence and development of HCC, RT-qPCR and western blotting were performed to analyze the expression levels of BTBD3 in HCC cell lines. The overexpression of the BTBD3 gene in various HCC cell lines indicated that BTBD3 may be a cancer-associated gene. The expression level of BTBD3 was highest in HepG2 cells; however, it was not in Bel7404 cells, as previously demonstrated by a nude mouse transplantation tumor with the Bel7404 cell line (21). To facilitate this study, the present study used Bel7404 cells for the in vitro experiments, including MTS and wound healing assays. Subsequently, the siRNA interference technique was used to investigate the effect of the BTBD3 gene on the proliferation and metastasis of Bel7407 cells. The results revealed there was a minimal impact on cell proliferation following silencing of the BTBD3 gene. However, significant inhibition of cell invasion $(\leq 50 \%)$ was demonstrated in the wound healing assay and the Transwell model. Based on the aforementioned findings, it may be concluded that the BTBD3 gene was overexpressed in HCC tissues and cell lines, which promoted the invasion and metastasis of cancer cells without affecting cell proliferation.

As there are limited previous studies regarding the function of the BTBD3 gene, this is the first study to demonstrate the promoting effect of the BTBD3 gene on HCC cell invasion; however, further confirmation by performing in vivo experiments, and investigation into the underlying mechanisms of BTBD3 in HCC cell migration and invasion, are required.

\section{Acknowledgements}

The present study was supported by the National Natural Science Foundation of China (grant no. 81272483) and Guangdong Natural Science Foundation (grant no. S2013010016728). 


\section{References}

1. Jemal A, Bray F, Center MM, Ferlay J, Ward E and Forman D: Global cancer statistics. CA Cancer J Clin 61: 69-90, 2011.

2. Siegel R, Ma J, Zou Z and Jemal A: Cancer statistics, 2014. CA Cancer J Clin 64: 9-29, 2014

3. Yan T, Zhao J, Bi X, Zhao H, Huang Z, Li ZY, Zhou JG, Li Y, Li C, Cai JQ and Zhao P: Prognosis of hepatocenular carcinoma: A study of 832 cases. Zhong Hua Zhong Liu Za Zhi 35: 54-58, 2013 (In Chinese).

4. Forner A, Llovet JM and Bruix J: Hepatocellular carcinoma. Lancet 379: 1245-1255, 2012.

5. Sherman M: Recurrence of hepatocellular carcinoma. N Engl J Med 359: 2045-2047, 2008.

6. Liu M, Jiang L and Guan XY: The genetic and epigenetic alterations in human hepatocellular carcinoma: A recent update. Protein Cell 5: 673-691, 2014.

7. Livak KJ and Schmittgen TD: Analysis of relative gene expression data using real-time quantitative PCR and the 2(-Delta Delta C(T)) method. Methods 25: 402-408, 2001.

8. Kishi Y, Hasegawa K, Sugawara Y and Kokudo N: Hepatocellular carcinoma: Current management and future development-improved outcomes with surgical resection. Int J Hepatol 2011: 728103, 2011

9. Shah SA, Cleary SP, Wei AC, Yang I, Taylor BR, Hemming AW, Langer B, Grant DR, Greig PD and Gallinger S: Recurrence after liver resection for hepatocellular carcinoma: Risk factors, treatment, and outcomes. Surgery 141: 330-339, 2007.

10. Roessler S, Jia HL, Budhu A, Forgues M, Ye QH, Lee JS Thorgeirsson SS, Sun Z, Tang ZY, Qin LX and Wang XW: A unique metastasis gene signature enables prediction of tumor relapse in early-stage hepatocellular carcinoma patients. Cancer Res 70: 10202-10212, 2010.

11. Fang Y, Xue JL, Shen Q, Chen J and Tian L: MicroRNA-7 inhibits tumor growth and metastasis by targeting the phosphoinositide 3-kinase/Akt pathway in hepatocellular carcinoma. Hepatology 55: 1852-1862, 2012.

12. Mizuguchi Y, Mishima T, Yokomuro S, Arima Y, Kawahigashi Y, Shigehara K, Kanda T, Yoshida H, Uchida E, Tajiri T and Takizawa T: Sequencing and bioinformatics-based analyses of the microRNA transcriptome in hepatitis B-related hepatocellular carcinoma. PLoS One 6: e15304, 2011.
13. Zhang P, Ma Y, Wang F, Yang J, Liu Z, Peng J and Qin H: Comprehensive gene and microRNA expression profiling reveals the crucial role of hsa-let-7i and its target genes in colorectal cancer metastasis. Mol Biol Rep 39: 1471-1478, 2012.

14. Stewart SE, Yu D, Scharf JM, Neale BM, Fagerness JA, Mathews CA, Arnold PD, Evans PD, Gamazon ER, Davis LK, et al: Genome-wide association study of obsessive-compulsive disorder. Mol Psychiatry 18: 788-798, 2013.

15. Schonrock N, Humphreys DT, Preiss T and Götz J: Target gene repression mediated by miRNAs miR-181c and miR-9 both of which are down-regulated by amyloid- $\beta$. J Mol Neurosci 46: 324-335, 2012.

16. Wu C, Xu B, Yuan P, Ott J, Guan Y, Liu Y, Liu Z, Shen Y, Yu D and Lin D: Genome-wide examination of genetic variants associated with response to platinum-based chemotherapy in patients with small-cell lung cancer. Pharmacogenet Genomics 20: 389-395, 2010.

17. Damgaard T, Knudsen LM, Dahl IM, Gimsing P, Lodahl M and Rasmussen T: Regulation of the CD56 promoter and its association with proliferation, anti-apoptosis and clinical factors in multiple myeloma. Leuk Lymphoma 50: 236-246, 2009.

18. Sud A, Del Bono EA, Haines JL and Wiggs JL: Fine mapping of the GLC1K juvenile primary open-angle glaucoma locus and exclusion of candidate genes. Mol Vis 14: 1319-1326, 2008.

19. Matsui A, Tran M, Yoshida AC, Kikuchi SS, U M, Ogawa M and Shimogori T: BTBD3 controls dendrite orientation toward active axons in mammalian neocortex. Science 342: 1114-1118, 2013.

20. Wang Q, Zhao Z, Shang J and Xia W: Targets and candidate agents for type 2 diabetes treatment with computational bioinformatics approach. J Diabetes Res 2014: 763936, 2014.

21. Chen Y, Lin MC, Yao H, Wang H, Zhang AQ, Yu J, Hui CK Lau GK, He ML, Sung J and Kung HF: Lentivirus-mediated RNA interference targeting enhancer of zeste homolog 2 inhibits hepatocellular carcinoma growth through down-regulation of stathmin. Hepatology 46: 200-208, 2007. 